\title{
Optimale Hautpflege beim Sport
}

- Sport hat auf die Haut eine ganze Reihe positiver Effekte. Laut Prof. Martina Kerscher, Leiterin des Lehrstuhls Kosmetikwissenschaft der Universität Hamburg, regt körperliche Aktivität die Durchblutung der Haut und damit den Metabolismus ihrer Zellen an. Durch die gesteigerte Stoffwechselaktivität erhöht sich die Kollagen- und Elastinproduktion. „Zudem können freie Radikale in der Epidermis verstärkt eliminiert werden“, erklärte Kerscher. Über die Schweißsekretion bilden sich weiterhin vermehrt bakterizide Dermocidine, was die Bakterienflora verbessert.

Allerdings belaste Sport auch die Haut, so Prof. Julia Welzel, Chefärztin der Klinik für Dermatologie und Allergologie, Klinikum Augsburg. Vor allem durch Schwitzen, mechanische Irritationen, häufiges Duschen und UV-Exposition bei Outdoor-Aktivitäten“. Für einen optimalen therapeutischen Index gilt es, , die positiven Wirkungen von Sport für die Haut zu nutzen und sie zugleich vor den Gefahren zu schützen“, betonte Kerscher. Dies erfordert eine Hautreinigung und -pflege, die allen voran den hauteigenen $\mathrm{pH}$-Wert erhält und die Barriere- und Regenerationsfunktionen fördert.

Diese Anforderungen erfüllen die Produkte von sebamed ${ }^{\circledR}$, wie experimentelle Hauttests und Anwendungsbeobachtungen - erst kürzlich unter Leitung von Welzer an 25 Sportlern - zeigen. Durch neue, nicht invasive Techniken wie die optische Kohärenztomographie und konfokale Lasermikroskopie, so Welzer, „konnten wir die exzellente Verträglichkeit und guten kosmetischen Eigenschaften nachweisen“ - etwa das lange Verbleiben des Schutzfilmes von sebamed Lotion ${ }^{\circledR}$.

bfro

Pressekonferenz „Sport - Strapazen oder Wohltat für die Haut“, Hamburg, 9. Juni 2010 (Veranstalter: Sebapharma)

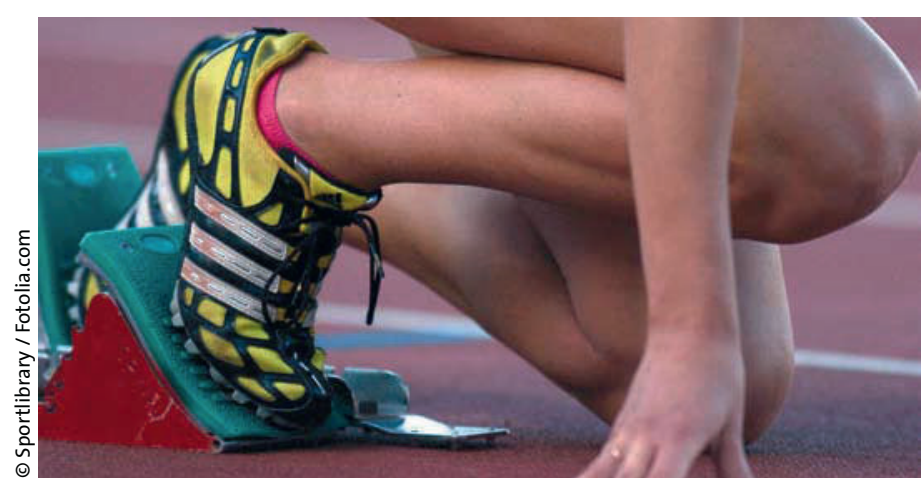

Erschwerte Bedingungen für die Hautbarriere...

\section{PDT-Preis erneut ausgeschrieben}

- Um die Forschung im Bereich der nicht-invasiven Behandlungsoptionen des hellen Hautkrebses zu unterstützen, wurde im Rahmen der 12. Jahrestagung der DWFA in Köln zum vierten Mal der PDT-Preis ausgeschrieben. Der Preis fördert Untersuchungen auf dem Gebiet der photodynamischen Therapie und der Fluoreszenzdiagnostik. Wegweisende Arbeiten aus diesem Bereich werden dabei von einem mit namhaften Wissenschaftlern besetzten Kuratorium prämiert. Initiator ist die Vereinigung Rheinisch-Westfälischer Dermatologen. Gestiftet wird der mit 5.000 Euro dotierte Preis vom Galderma Förderkreis e. V.

Voraussetzung für die Teilnahme am Wettbewerb ist, dass die Arbeit zum Zeitpunkt der Abgabe jünger als drei Jahre und bereits publiziert oder zur Publikation angenommen ist. Die Bewerber sollten deutschsprachig oder an einer deutschsprachigen Institution tätig sein. Einsendeschluss ist der 30. August 2010. Die Teilnahmebedingungen sind beim Galderma Förderkreis e. V., Georg-Glock-Str. 8, 40474 Düsseldorf, erhältlich.

Nach Informationen von Galderma

\section{Wundmanagement: Therapie und Pflege erleichtern}

- Mehr denn je sind heute innovative Konzepte für eine moderne Wundversorgung gefragt. Neben besserer Koordination der verschiedenen therapeutischen und pflegerischen Aktivitäten wird der Einsatz moderner Wundauflagen empfohlen. Mit UrgoCell ${ }^{\circledR}$ Lite und UrgoCell ${ }^{\circledR}$ Adhesive Contact sind seit kurzem zwei hydroaktive Wundauflagen in Deutschland verfügbar. Sie dürften nach Meinung der Experten die therapeutischen und pflegerischen Möglichkeiten bei chronischen Wunden erheblich erweitern, weil sie alle Vorteile der feuchten Wundbehandlung bieten.
Wie Dr. Hendrik Altenkämper aus Plettenberg erläuterte, bildet die Matrix dieser Wundauflagen bei Kontakt mit dem Wundsekret einen Lipidokolloidfilm. Dieser schafft zwischen Wunde und Auflage ein dauerhaft feuchtes Wundmilieu. Die einsetzende Gelbildung schont den gesamten Wundbereich und ermöglicht einen schmerzfreien, patientengerechten Verbandswechsel, ohne die zumeist sehr empfindlichen, nachwachsenden Gewebestrukturen zu beeinträchtigen.

Aktuelle klinische Studienergebnisse zur weiterentwickelten Wundauflage Urgotül ${ }^{\circledR}$ Soft präsentierte Dr. Sylvie Meaume aus
Irvy sur Seine/Frankreich. In der Studie an 44 Patienten erfolgte eine wöchentliche Beurteilung durch den Prüfarzt, außerdem wurde die Akzeptanz der Behandlung bei Pflegepersonal und Patienten erfasst. Nach vier Wochen Behandlung konnte eine Oberflächenreduktion bei chronischen Wunden von $41,9 \%$ und akuten Wunden von $78,2 \%$ gezeigt werden.

Jset

Launch-Pressekonferenz „UrgoCell ${ }^{\circledR}$ Lite und UrgoCell ${ }^{\circledR}$ Adhesive Contact”, Hannover, 24. März 2010 (Veranstalter: Urgo) 